Ref.TH. 3268-CERN

\title{
FINITENESS, 1/N EXPANSION AND SUPERSYMMETRY BREAKING
}

\author{
A.C. Davis, P. Salomonson
}

and

J.W. van Holten

CERN -- Geneva

\begin{abstract}
The possibility of spontaneous supersymmetry breaking in the limit of large $\mathrm{N}$, in models with an internal $O(N)$ symmetry, is demonstrated by constructing an explicit example in two dimensions. The model is finite and this is shown to be important for the supersymmetry breaking. A general criterion for finiteness of scalar superfield theories in two dimensions is given. Finally the generalization of our results to three dimensions, and their relevance to four-dimensional models, is discussed.
\end{abstract}

Ref.TH. 3268-CERN

23 March 1982 


\section{1. - INTRODUCTION}

Supersymmetric field theories are theories which possess a symmetry in the Lagrangian between fermions and bosons. They have several attractive features. For instance, in supersymmetric theories there are many cancellations between divergences from boson and fermion loops ${ }^{1 /, 2)}$. It has even been conjectured that $N=4$ supersymmetric Yang-Mills ${ }^{2), 3)}$ and $N=8$ supergravity ${ }^{21,4)}$ are finite to all orders in perturbation theory. The softening of divergences in supersymmetric theories has been applied in physics to help solve diverse problems like the non-renormalizability of quantum gravity ${ }^{4)}$ and the gauge hierarchy problem ${ }^{5}$. Indeed, it seems likely that unified field theories which involve gravity will have to possess supersymmetry; for such a theory $N=8$ supergravity is the most likely candidate.

Whilst supersymmetry is theoretically attractive, it is not a manifest symmetry of Nature. This necessitates the establishment of a realistic mechanism of supersymmetry breaking for these theories. Supersymmetry breaking is not induced by radiative corrections in four-dimensional gauge theories 61,7$)$ and, therefore, must be present at the tree level or be induced by non-perturbative effects like instantons ${ }^{8}$ ) or large $\mathrm{N}$ behaviour ${ }^{9)}$. Unfortunately, the only known fourdimensional example of supersymmetry breaking in the large $N$ limit is plagued with pathologies ${ }^{10)}$. The non-perturbative generation of condensates could lead to supersymmetry breaking and such a mechanism also seems particularly relevant to supergravity theories. In supergravity the difficulties of supersymmetry breaking are complicated. The possibility of a supersymmetric Higgs effect has been established only for $\mathrm{N}=1$ supergravity ${ }^{11}$ ). For extended supergravity a mechanism for generating masses by dimensional reduction has been found $\left.{ }^{4}, 12\right)$. However, these theories do not possess the usual local supersymmetry. Because of the non-linearity of the scalar potential and the necessity of producing a particle spectrum at low energy that is very different from that of the elementary fields, one may expect supersymmetry breaking to take place by non-perturbative effects. Indeed, i.t has been conjectured that SU(5) grand unification is obtained from $N=8$ supergravity below the Planck mass ${ }^{13)}$. This scenario requires the SU(5) spectrum to be generated as bound states from the fundamental $N=8$ fields. other scenarios, for example with gauged so(8) symmetry ${ }^{14}$, require confinement of the elementary fields.

Whilst dynamical generation of bound states is known to happen in some nonlinear models $\left.\left.{ }^{15}\right), 16\right)$, a puzzling aspect of this envisaged scenario is that $N=8$ supergravity is presumably finite. It might be wondered whether quantum corrections could still induce such important effects as the ones described here. We address this question in this paper. 
To explore non-perturbative phenomena, we use the $1 / \mathrm{N}$ expansion; this is usually appropriate for theories with large internal symmetry groups. We show that supersymmetry can be broken in the large $N$ limit of finite, supersymmetric field theories with an $O(N)$ internal symmetry. Indeed, we have shown that in an $O\left(N_{1}\right) \times O\left(N_{2}\right)$ generalization of the non-linear sigma model, finite in the large $N$ limit, supersymmetry is spontaneously broken and the supermultiplets of Lagrange mulitipliers acquire dynamics. This result, which has been presented elsewhere ${ }^{17)}$, is encouraging for unification schemes based on $N=8$ supergravity.

To find specific finite models, we turn our attention to two-dimensional theories with scalar supermultiplets. Whilst such models are unrealistic for Nature, they have proved useful in the past in gaining intuition for more complex, four-dimensional theories. They are also amenable to calculational techniques and the demonstration of specific effects, desirable in more realistic models.

The plan of this paper is as follows. In Section 2 we discuss the general formalism for supersymmetric theories. We discuss general requirements for supersymmetry breaking and give consistency conditions for the effective potential. We then give a general criterion for the finiteness of two-dimensional supersymmetric theories with scalar supermultiplets. In Section 3 we discuss finiteness versus supersymmetry breaking. We do this in the context of the supersymetric non-linear sigma model, showing how infinite renormalization actually leads to supersymmetry restoration in this model.

As a consequence, in Section 4 we turn our attention to a model which is finite to all orders in perturbation theory and is $1 / \mathrm{N}$ expandable. This model exhibits supersymmetry breaking in the large $N$ limit. Section 5 contains a summary of our results. We also extend the analysis beyond two dimensions, showing that supersymmetry is also broken in the three-dimensional version of our model. Some comments are made about the situation in four dimensions.

\section{2. - FINITE MODELS IN TWO DIMENSIONS}

a) General Formalism ${ }^{18)}$

Supersymmetric scalar field theories in dimension $\mathrm{n}=1,2$ or 3 describe one or more scalar supermultiplets, $\Phi \equiv(\phi, \mathrm{U}, \mathrm{F})$, consisting of real scalars $(\phi, F)$ of dimension $((n / 2)-1, n / 2)$ respectively and a Majorana spinor of dimension $((n / 2)-1 / 2)$. Their transformation properties are: 


$$
\begin{aligned}
& \delta \varphi=\bar{\epsilon} u \\
& \delta u=(\phi \varphi+F) \epsilon \\
& \delta F=\bar{\epsilon} \phi u
\end{aligned}
$$

where $\varepsilon$ is the anticommuting Majorana spinor parameter of supersymmetry. Due to the inclusion of the field $F$ the supersymmetry algebra

$$
\left[\delta\left(\epsilon_{2}\right), \delta\left(\epsilon_{1}\right)\right]=\left(2 \bar{\epsilon}_{2} \gamma_{r} \epsilon_{1}\right) \partial_{\mu}
$$

is realized independently of the dynamics, i.e., of the equations of motion. It is possible to construct a large number of invariants from these fields. However, in most cases we restrict ourselves to theories with the usual type of kinetic terms. Thus, the possible Lagrangians are as follows:

$$
\mathcal{L}=L_{\text {kin }}+L_{\text {int }}
$$

where the kinetic part is

$$
L_{k \text { in }}=-\frac{1}{2}\left(\partial_{\mu} \varphi_{i}\right)^{2}-\frac{1}{2} \bar{u}_{i} \not u_{i}+\frac{1}{2} F_{i}^{2}
$$

and the interaction terms are

$$
L_{\text {int }}=-F_{i} v_{o i}^{\prime}+\frac{1}{2} v_{0 i j}^{\prime \prime} \bar{u}_{i} u_{j}
$$

where $V_{o i}^{\prime}$ and $V_{o i j}^{\prime \prime}$ are the first and second derivatives of an arbitrary function $V\left[\phi_{i}\right]$ of the scalars $\phi_{i}(i=1,2, \ldots)$. This is the superpotential. Since the $F_{i}$ fields have no kinetic terms, they are auxiliary fields. In principle, they can be eliminated by their equation of motion

$$
F_{i}=v_{0}^{\prime}
$$

though this is not always advantageous.

The quantum corrections to such models are easily evaluated, giving the oneloop effective potential to be

$$
\begin{aligned}
\bar{V}_{0+1}(F, \varphi)=-\frac{1}{2} F^{2}+F V^{\prime}(\varphi)+\frac{1}{2} \int \frac{d^{n} p}{(2 \pi)^{n}} & {\left[\operatorname{Tr} \log \left(p^{2}+V^{\prime \prime 2}+V^{\prime \prime \prime} F\right)(2.7)\right.} \\
& \left.-\operatorname{Tr} \log \left(p^{2}+V^{\prime \prime 2}\right)\right]
\end{aligned}
$$


where $\mathrm{n}$ is the number of dimensions. Again, the auxiliary fields can be eliminated using the equations of motion,

$$
0=\frac{\partial V}{\partial F}=-F+U^{\prime}+\frac{1}{2} \int \frac{d^{n} p}{(2 \pi)^{n}} \operatorname{Tr}\left[\left(p^{2}+V^{\prime \prime 2}+U^{\prime \prime \prime} F\right)^{-1} U^{m}\right]
$$

giving the solution $F(\phi)$. Substituting for $F$ the effective potential is obtained as a function of the physical scalar fields.

$$
V_{o+1}(\varphi)=\bar{V}_{o+1}(F(\varphi), \varphi)
$$

Actually, (2.9) contains terms beyond one loop. Inserting the appropriate factors of $\hbar$ into (2.7) - (2.9), arbitrary high powers of $\hbar$ will, in general, then appear in (2.9). In a strict one-10op approximation, terms of order $\hbar^{2}$ and higher are neglected. However, in $1 / \mathrm{N}$ expandable models the full expression in (2.9) is useful since it contains all the leading order contributions to the effective potential. This is because the auxiliary fields in these supersymmetric theories also serve to linearize the interactions for the $O(N)$ fields. Of course, the actual technique of the $1 / \mathrm{N}$ expansion is a little different from the loop expansion, but our general comments are equally applicable.

It is easy to prove that, for real values of the potential (2.9), there is at most one branch, that $V$ is positive and zero only for vaiues of $\phi$ such that $F(\phi)$ is zero. The proof is as follows. The second derivative of $\bar{V}$ with respect to the auxiliary fields defines a quadratic form,

$$
\begin{aligned}
& X_{a} \frac{\partial^{2} \bar{V}_{o+1}(F, \varphi)}{\partial F_{a} \partial F_{b}} X_{b} \\
& =-x^{2}-\frac{1}{2} \int \frac{d_{p}^{n}}{(2 \pi)^{n}} \operatorname{Tr}\left(M^{-\frac{1}{2}} V^{\prime \prime \prime} \times M^{-\frac{1}{2}}\right)^{2},
\end{aligned}
$$

where

$$
M=p^{2}+V^{\prime \prime 2}+V^{\prime \prime \prime} F \text {. }
$$

We have suppressed the supermultiplet indices. Since $M$ is a symmetric matrix, and should be positive, there is no problem in taking the square root. (For large enough $|F| M$ can have negative eigenvalues, but then $V$ will not be real.) 
Now, (2.10) is negative definite. Thus, the second derivatives of $\bar{v}$ with respect to the auxiliary fields form a negative definite matrix. This means that $\bar{V}$ as a function of the auxiliary fields can have at most one stationary point. This is a maximum. Thus, $\partial \bar{V} / \partial F=0$ has, at most, one solution for any given $\phi$; this solution corresponds to a maximum of $\bar{V}$. Since $\bar{V}(F=0, \phi)=0, \bar{V}$ must be positive at this maximum, unless $F(\phi)=0$ in which case $\bar{V}=0$.

The criterion for unbroken supersymmetry will be that the solution $F(\phi)$ of (2.8) is zero for some value of $\phi$.

b) Finiteness

From (2.7) the one-loop corrections to the effective action are

$$
\begin{aligned}
& -\frac{1}{2} \operatorname{Tr} \log \left(1+\frac{1}{-\square+v^{\prime \prime 2}} F \cdot V^{\prime \prime \prime}\right) \\
& =-\frac{1}{2} \operatorname{Tr}\left(\frac{1}{-\square+v^{\prime \prime 2}} F \cdot V^{\prime \prime \prime}\right)+O\left(F^{2}\right),
\end{aligned}
$$

where the terms $O\left(F^{2}\right)$ are all finite. Hence the linear $F$ term is the only divergent one. Thus, a necessary condition for the theory to be finite at one-1oop level is

$$
F . V^{\prime \prime \prime}=0 \quad \text { or } \operatorname{Tr} V^{\prime \prime}=\text { constant. }
$$

We show that this condition suffices to make the theory ultra-violet finite, in two dimensions, to all orders in perturbation theory ${ }^{19)}$.

The proof is as follows. We define the propagators for the fields $\phi_{i}, u_{i}$ and $F_{i}$ to be those obtained from $\ell_{k i n}$ only. Thus, mass terms resulting from $\ell_{\text {int }}$ are treated as vertices. Since we are only interested in the large $p^{2}$ behaviour, this is no problem. With the notation of Fig. 1 these propagators can be written as 


$$
\begin{aligned}
\left\langle\varphi_{i} \varphi_{j}\right\rangle & =\frac{\delta_{i j}}{p^{2}}, \\
\left\langle u_{i} \bar{u}_{j}\right\rangle & =\frac{i \frac{p \delta_{i j}}{p^{2}},}{} \\
\left\langle F_{i} F_{j}\right\rangle & =-\frac{p^{2}}{p^{2}} \delta_{i j}=-\delta: j .
\end{aligned}
$$

Firstly, consider diagrams with just scalar fields. As given by power counting, the degree of divergence is

$$
D=2(L-P),
$$

where $L$ is the number of loop integrals and $P$ the number of propagators. (These contribute $+2,-2$, respectively.) Now, the number of loop integrals is

$$
\text { L } \quad=P-V+1 \text {, }
$$

where $\mathrm{V}$ is the number of vertices. Combining (2.15) and (2.16) we obtain

$$
D \quad=2(1-v)
$$

This is non-negative only for $V=0$ or 1 .

Next, consider the inclusion of auxiliary fields and fermions. Note that each vertex can be attached to, at most, one auxiliary field propagator, or two fermion lines. An auxiliary field contributes $p^{2}$, a fermion $\not$ to the numerator. Fermion lines closing on a single vertex do not contribute because they are proportional to $V_{0 k k . . .}^{\prime \prime . .}$, where the number of derivatives depends on the number of scalar fields attached to the vertex. With the exception of the vacuum energy density diagram, which is a constant, these diagrams vanish using (2.13). Hence, auxiliary fields and fermion lines always connect two different vertices. Thus we deduce that the degree of divergence of an arbitrary diagram is

$$
D \leq 2\left(1-v+2\left[\frac{V}{2}\right]\right)
$$


where $\left[\frac{V}{2}\right]$ is the integer part of $V / 2$. Therefore, diagrams with internal fermion or auxiliary field lines can only be divergent if $V=0,1,2$.

Finally, we consider the various cases in detail. Diagrams with no external lines ( $\mathrm{V}=0$ or $\mathrm{V}=1$ for mass insertions) contribute only to the vacuum energy density. This is either zero or a positive constant, depending on whether or not supersymmetry is broken.

Now, all other diagrams with $V=1$, as shown in Fig. 2, are proportional to

$$
\int \frac{d^{2} p}{(2 \pi)^{2}} \frac{1}{p^{2}} V_{o k k}^{\prime \prime} \ldots . . .=0
$$

vanishing because of (2.13). We are now left with diagrams with two vertices and internal auxiliary field or fermion lines, as shown in Fig. 3. Taking into account combinatoric factors, we obtain

$$
\begin{aligned}
& -\frac{1}{2 n !} \frac{n(n-1)}{2} V_{\cdots}^{(n)} U_{\ldots}^{(n)} \int \frac{d^{2} p_{i}}{(2 \pi)^{2}} \delta^{(2)}\left(p_{1}+\cdots+p_{n}\right) T r \frac{p_{1} p_{2}}{p_{1}^{2}+\cdots+p_{n}^{2}} \\
& +\frac{1}{2 n !} n V^{(n)} V^{(n)} \cdot \int_{\frac{d^{2}}{(2 \pi)^{2}}} \delta^{(2)}\left(p_{1}+\cdots+p_{n}\right) \frac{-p^{2}}{p_{1}^{2}+\cdots+p_{n}^{2}}
\end{aligned}
$$

For simplicity we have taken the external momenta to vanish; they would only give finite extra contributions. The integral in (2.20) is zero because one may make the replacement

$$
n(n-1) p_{1} \cdot p_{2}+n p_{1}^{2}=\left(p_{1}+\cdots+p_{n}\right)^{2}
$$

which vanishes due to momentum conservation.

Since all diagrams with external fermion lines are finite, we have neglected them in this analysis. Thus, (2.13) guarantees the finiteness of our models to all orders in perturbation theory. Similar considerations hold for $1 / \mathrm{N}$ expandable models. 


\section{3. - THE 1/N EXPANSION AND SUPERSYMMETRY BREAKING IN RENORMALIZABLE MODELS}

Radiative corrections do not induce supersymmetry breaking either in fourdimensional scalar or gauge models ${ }^{6), 7)}$. As explained in the Introduction, it has not been possible to find a Higgs mechanism in extended supergravity to break supersymmetry and give a mass to the gravitinos. Though it can be done by dimensional reduction, there are reasons to believe that realistic supersymmetry breaking mechanisms might be of a non-perturbative nature. For example, the generation of mass terms alone is not sufficient to obtain a realistic particle spectrum. A useful method for studying the non-perturbative behaviour of quantum systems especially in relation to the generation of bound states, is through the $1 / \mathrm{N}$ expansion. This technique is applicable in most of the models considered below and we will use it extensively.

Before proceeding, it is useful to list some characteristics of broken global supersymmetry ${ }^{20}$ ). These include:

- the auxiliary field $F$ acquires a non-zero vacuum expectation value. In two dimensions this can only happen for auxilary fields which are singlets under the internal symmetry. An expectation value of a non-singlet would break the internal symmetry, in contradiction with coleman's theorem ${ }^{21}$ )

- the vacuum energy density is non-zero and positive. In fact

$$
\epsilon=\frac{1}{2}\langle F\rangle^{2}
$$

hence this criterion follows from the previous one

- there is a massless Goldstone fermion. Since this fermion belongs to the same multiplet as the auxiliary field, which acquires the expectation value, it is also a singlet under the internal symetry. This condition is also related to the previous ones because $\langle F\rangle$ represents the coupling constant of the Goldstone fermion to the supersymmetry current.

One aim of this paper is to contrast the situation as regards the spontaneous breaking of supersymmetry in models which are finite and ones which require infinite renormalizations. In this section, we will therefore consider a model which is not completely finite but has in the large $N$ limit one finite counterterm. This counterterm is linear in the auxiliary field and has a direct effect upon the breaking of supersymmetry. In fact we will show that supersymmetry is restored by the inclusion of this counterterm. In the next section we will then show that in a model where this counterterm is absent, supersymmetry can indeed be broken. 
However, we do not claim that finiteness is a necessary condition for spontaneous supersymmetry breaking. This is illustrated by an example we discuss at the end of this section. The first model consists of an $O(N)$ scalar multiplet $\Phi_{i}=\left(\Phi_{i}, v_{i}, F_{i}\right)$ and a singlet scalar multiplet $\Phi=(s, u, D)$. The Lagrangian is defined by the superpotential

$$
V=\frac{9}{2 \sqrt{N}} S\left(\varphi_{i}^{2}+N \alpha\right)
$$

We have already included the appropriate factors of $\mathrm{N}$. This gives

$$
\begin{aligned}
\mathcal{L} & =-\frac{1}{2}\left(\partial_{N} s\right)^{2}-\frac{1}{2} \bar{u} \phi u+\frac{1}{2} D^{2}-\frac{1}{2}\left(\partial_{N} \varphi_{i}\right)^{2} \\
& -\frac{1}{2} \bar{v}_{i} \partial v_{i}+\frac{1}{2} F_{i}^{2}-\frac{g}{2} \alpha \sqrt{N} D-\frac{g}{2 \sqrt{N}} D \varphi_{i}^{2}-\frac{g}{\sqrt{N}} s \varphi_{i} F_{i} \\
& +\frac{g}{\sqrt{N}} \varphi_{i} \bar{v}_{i} u+\frac{g}{2 \sqrt{N}} s \bar{v}_{i} v_{i} .
\end{aligned}
$$

Integrating out the $O(N)$ fields we obtain the effective action

$$
\begin{aligned}
& \left.S_{e f f}=\int d^{2} \times\left[-\frac{1}{2}(\partial N s)^{2}-\frac{1}{2} \bar{u}\right\rangle u+\frac{1}{2} D^{2}-g \alpha \sqrt{N D}\right] \\
& -\frac{N}{2} \operatorname{Tr} \log \left[-D+\frac{g}{\sqrt{N}} D+\frac{g^{2}}{N}\left(s^{2}+\bar{u}\left(-\not+\frac{9 s}{\sqrt{N}}\right)^{-1} u\right)\right] \\
& +\frac{N}{2} \operatorname{Tr} \log \left(-\not+\frac{g s}{\sqrt{N}}\right) \text {. }
\end{aligned}
$$

The fields $s$ and $D$ can be written as

$$
\begin{aligned}
& s=\sqrt{N} \bar{s}+\sigma, \\
& D=\sqrt{N} \bar{D}+\Delta,
\end{aligned}
$$

where $\bar{s}$ and $\bar{D}$ are the vacuum expectation values, to be determined later, and $\sigma$ and $\Delta$ are the fluctuations.

Collecting together the $O(N)$ terms we obtain the vacuum energy density,

$$
E=N\left[-\frac{1}{2} \bar{D}^{2}+9 \alpha \bar{D}+\frac{1}{2} \operatorname{Tr} \log \left(1+\frac{9}{-\square+9^{2} \bar{s}^{2}} \bar{D}\right)\right] \text {. }
$$

Clearly, this is zero for $\bar{D}=0$, i.e., when supersymmetry is unbroken. 
The terms $O(\sqrt{\mathrm{N}})$ are linear in the fluctuations $\sigma$ and $\Delta$ and thus give the saddle-point conditions. These are

$$
\begin{gathered}
\bar{D}-g \alpha-\frac{g}{2} \int \frac{d^{2} p}{(2 \pi)^{2}} \frac{1}{p^{2}+g \bar{D}+g^{2} \bar{s}^{2}}=0 \\
g^{2} \bar{s}^{2} \int \frac{d^{2} p}{(2 \pi)^{2}}\left(\frac{1}{p^{2}+g \bar{D}+g^{2} \bar{s}^{2}}-\frac{1}{p^{2}+g^{2} \bar{s}^{2}}\right)=0
\end{gathered}
$$

These equations determine $\overline{\mathrm{D}}$ and $\overline{\mathrm{s}}$. Equation (3.7) contains a divergent integral, which we will discuss shortly. Equation $(3.8)$ is finite and can be written as

$$
\bar{s} \log \left(1+\frac{\bar{D}}{g \bar{s}^{2}}\right)=0,
$$

which admits two solutions: $\bar{D}=0$ and supersymmetry is unbroken; $\bar{s}=0$, in which case supersymmetry may be broken if $\overline{\mathrm{D}} \neq 0$. Which solution is actually realized depends on the solution to (3.7). Suppose there is a way to control the divergence of the integral, i.e., a physical uitra-violet cut-off. Then, if $\alpha$ is positive, the solution $\bar{D}=0$ is impossible, supersymmetry is broken and $\bar{s}=0$ from the second equation. In this case, $u$ is a Goldstone fermion of broken supersymmetry, whilst the $O(N)$ scalar fields acquire a mass gD. The $O(N)$ fermions remain massless.

However, if the cut-off is removed, the situation changes drastically. Using dimensional regularization $(3.7)$ becomes

$$
\frac{1}{9} \bar{D}=\alpha-\frac{1}{4 \pi} \frac{1}{d-2}-\frac{1}{8 \pi} \log \left(\frac{9 \bar{D}+g^{2} \bar{s}^{2}}{4 \pi \mu_{0}^{2}}\right)
$$

where $\mu_{0}$ is the scale parameter, defining the unit of mass. To renormalize an infinite counterterm for $\alpha$ is necessary

$$
\alpha\left(\mu^{2}\right)=\frac{1}{4 \pi(d-2)}+\frac{1}{8 \pi} \log \left(\frac{\mu^{2}}{4 \pi \mu_{0}^{2}}\right)
$$

Note that the finite part of $\alpha$, parametrized by the variable $\mu^{2}$, can be positive, negative or zero, depending on $\mu^{2}$. Combining (3.10) and (3.11) we obtain 


$$
\frac{1}{9} \bar{D}=-\frac{1}{8 T} \log \left(\frac{9 \bar{D}+9^{2} \bar{s}^{2}}{\mu^{2}}\right) \text {. }
$$

Hence, for any $\mu$, i.e., for any value of the finite part of $\alpha$, the solution $\overline{\mathrm{D}}=0$ exists, with

$$
g^{2} \bar{s}^{2}=\mu^{2}
$$

As the supersymetric solution has zero vacuum energy and the Hamiltonian is positive definite, this solution is the absolute minimum of the potential and is thus the one realized by the system. Since the linear $D$ term is, by itself, invariant, the renormalization of $\alpha$ respects supersymmetry. The generated mass for the $O(N)$ multiplet is

$$
m^{2} \Phi_{i}=g^{2} \bar{s}^{2}=\mu^{2},
$$

i.e., identical for the bosons and fermions.

Finally, the $O(1)$ terms in eff give the masses and wave function renormalization for the singlet field. Both are finite and we obtain

$$
\begin{aligned}
L_{e f f}= & -\frac{1}{2}\left(\partial_{\mu} \sigma\right)^{2}-\frac{1}{2} \bar{u} u+\frac{1}{2} \Delta^{2} \\
+ & \operatorname{Tr}_{r}\left[\frac{g^{2}}{4}\left(\frac{1}{-\square+\mu^{2}} \Delta\right)^{2}+\mu g^{2}\left(\frac{1}{-\square+\mu^{2}}\right) \Delta\left(\frac{1}{-\square+\mu^{2}}\right) \sigma\right. \\
& \left.-\frac{g}{2}\left(\frac{\partial_{\mu} \sigma}{-\square+\mu^{2}}\right)^{2}-\frac{g^{2}}{2}\left(\frac{1}{-\square+\mu^{2}}\right) \bar{u}\left(\frac{\partial+\mu}{-\square+\mu^{2}}\right) u\right] .
\end{aligned}
$$

Clearly, kinetic terms for $\sigma, u$ and $\Delta$ are induced by the quantum corrections; here they enter as a wave function renormalization for the entire singlet supermultiplet. In the limit $\mathrm{p}^{2} \rightarrow 0$ we obtain

$$
7^{2}(0)=1+\frac{9^{2}}{8 \pi r^{2}}
$$

giving the mass of the $O(N)$ singlet fields to be 


$$
m_{\text {singlet }}^{2}=\frac{1}{z^{2}(0)}\left(\frac{g^{2}}{4 \pi r}\right)^{2}
$$

All other contributions to $\mathcal{L}_{\text {eff }}$ vanish in the large $N$ limit.

That the entire singlet supermultiplet receives wave function renormalization suggests that we could have omitted the kinetic terms for the singlet fields completely in (3.3). They would then play the role of Lagrange multipliers, imposing the constraints,

$$
\begin{aligned}
\varphi_{i}^{2}+N \alpha & =0, \\
\varphi_{i} F_{i}-\frac{1}{2} \bar{\nu}_{i} v_{i} & =0,
\end{aligned}
$$

and our model would reduce to the supersymmetric $O(N)$ non-linear sigma model. As first noted by Alvarez ${ }^{16}$, kinetic terms are then induced for the scalar multiplet by the quantum corrections, unlike the case for the usual non-linear sigma model.

Thus, we have shown that, in the large $N$ limit, our model is finite up to a renormalization of $\alpha$. This renormalization restores supersymmetry. The singlet multiplet becomes dynamical via quantum corrections.

In spite of the above example, it would be wrong to infer that infinite renormalizations always spoil supersymmetry breaking. To show this, it is instructive to consider another theory with broken supersymmetry. It is the supersymmetric version of $\phi^{4}$ theory, with a single superfield and a superpotential given by

$$
V(\varphi)=\frac{1}{6} 9\left(\varphi^{3}-3 c \varphi\right)
$$

The one-dimensional (quantum mechanical) version of this theory was studied in Ref. 8). At the tree level, supersymmetry is unbroken if $c \geq 0$. However, it was found that supersymmetry was always broken in the quantum theory, though, for large $c$, only by tiny instanton effects.

The one-loop effective potential is easily calculated. From (2.8) we see that the condition for unbroken supersymmetry is that there is a solution to the equation 


$$
\varphi^{2}-c+\int \frac{d^{n} p}{(2 \pi)^{n}} \quad \frac{1}{p^{2}+g^{2} \varphi^{2}}=0
$$

In the Euclidean case, the integral is positive definite. Thus the one-loop condition for supersymmetry is clearly more restrictive than the tree condition. For $\mathrm{n}=1$, the integral is finite and we obtain

$$
\phi^{2}=<-\frac{1}{2|9 \varphi|}
$$

If now

$$
c<\frac{3}{|49|^{2 / 3}}
$$

Eq. (3.21) has no solution. Consequently, for $0 \leq c<3 /|4 g|^{2 / 3}$, supersymmetry is broken by the one-loop corrections.

In principle, the situation in two dimensions is similar ${ }^{71,18)}$. From (3.20) it follows that quantum corrections tend to break supersymmetry. However, for $\mathrm{n}=2$ the integral is divergent and as a result we have to renormalize $c$. After renormalization, $c$ depends on the scale at which it is defined:

$$
\varphi^{2}=c(\mu)+\frac{1}{4 \pi} \log \left(\frac{g^{2} \phi^{2}}{r^{2}}\right)
$$

and

$$
c\left(\mu^{\prime}\right)=c(\mu)+\frac{1}{4 \pi} \log \left(\frac{\mu^{\prime 2}}{\mu^{2}}\right)
$$

of course, the scale dependence cancels in (3.23). Whether or not supersymmetry is broken does not depend on the renormalization scale chosen. However, we find it difficult to claim that supersymetry is broken in this model by radiative corrections, since this involves a comparison between the classical model and the quantum model, with different kinds of parameters.- a constant $c$ and a scaledependent $\left.c(\mu)^{*}\right)$. It is an advantage of finite models that this complication is absent and the origin of spontaneous supersymmetry breaking can be more

*T could still be claimed that radiative corrections break supersymetry here, if it were broken for all values of $c(\mu)$. This might be true if one could sum the perturbation series completely. The one-ioop corrections, however, still admit a range of values for which supersymmetry is not broken. 
clearly assessed. In the next section we will present such a finite, twodimensional model and show that it exhibits supersymmetry breaking at the quantum level.

\section{4. - SUPERSYMMETRY BREAKING IN A FINITE MODEL}

In Section 3 we have seen that it may be possible to break supersymmetry by quantum corrections in two-dimensional modeis, but that it is difficult to decide this without summing the whole perturbation series. On the other hand, in the model where we could get information about the exact results using the $1 / \mathrm{N}$ expansion, supersymmetry turned out to remain unbroken because of the infinite counterterm which had to be introduced.

Therefore it is worth while to construct a model which is both $1 / \mathrm{N}$ expandable and finite. Then one can hope to establish supersymmetry breaking unambiguously, at least in the large $N$ limit. We will present such a model.

Our model contains only scalar supermultiplets and possesses an $O\left(N_{1}\right) \times O\left(N_{2}\right)$ internal symmetry. The supermultiplets are as follows: $\left(\vec{\phi}_{1}, \vec{v}_{2}, \vec{F}_{1}\right)$ and

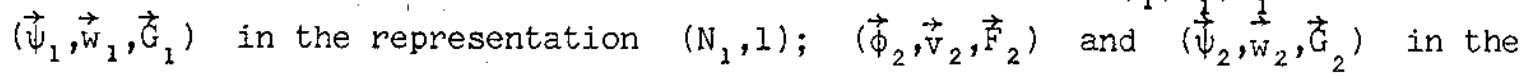
representation $\left(I, N_{2}\right)$; and finally $(S, u, D)$ in the singlet representation $(1,1)$. The Lagrangian is defined by the superpotential

$$
V\left(\varphi_{\nu}, \Psi_{\nu}, s\right)=\sum_{\nu=1,2} g_{\nu}\left(m \vec{\varphi}_{\nu} \cdot \vec{\psi}_{\nu}+\frac{1}{2} s \vec{\varphi}_{\nu}^{2}\right) .
$$

If we impose the condition

$$
\sum_{\nu} g_{\nu} N_{\nu}=0
$$

the superpotential satisfies the criteria for finiteness of the theory in two dimensions, given in Section 2. We can implement (4.2) by defining

$$
\begin{aligned}
& N_{\nu}=n_{\nu} N, \\
& g_{\nu}=(-1)^{\nu} \frac{g}{n_{\nu}} .
\end{aligned}
$$

Then a $1 / N$ expansion can be defined, and this leaves the ratio $N_{1} / N_{2}=-g_{2} / g_{1}$ fixed.

This model, but without kinetic terms for the singlet fields, has been discussed in detail elsewhere ${ }^{17)}$. Here we wish to present a more qualitative discussion, based on the results obtained there. An effective action for the singlet 
fields can be found by integrating over the $O(N)$ vector fields, taking into account the fact that they do not develop vacuum expectations, in agreement with Coleman's theorem. As a result, the effective potential in the limit $q^{2} \rightarrow 0$ (i.e., for constant fields) depends only on the vacuum expectation values of the fields $s$ and $D$.

To be more precise, the effective Lagrangian reads

$$
\begin{aligned}
L_{e f f}= & -\frac{1}{2}\left(\partial_{r} s\right)^{2}-\frac{1}{2} \bar{u} \phi u+\frac{1}{2} D^{2} \\
& -\frac{N}{2} \sum_{\nu} n_{\nu} \operatorname{Tr}_{r} \int \frac{d^{2} q}{(2 \pi)^{2}}\left[\log B\left(q^{2}\right)-\log F\left(q^{2}\right)\right]
\end{aligned}
$$

where

$$
\begin{aligned}
& B\left(q^{2}\right)=\left(\begin{array}{c}
q^{2}+m^{2} g_{\nu}^{2}+g_{\nu} D+g_{\nu}^{2}\left(s^{2}+\bar{u}\left(\not \partial-g_{\nu} s-m^{2} g_{\nu} \frac{q}{\square}\right)^{-1} u\right. \\
m g_{\nu}^{2} s
\end{array}\right. \\
& F\left(q^{2}\right)=\left(\begin{array}{cc}
q^{2}+m^{2} g_{\nu}^{2}+g_{\nu}^{2} s^{2} & m g_{\nu}^{2} s \\
m g_{\nu}^{2} s & q^{2}+m^{2} g_{\nu}^{2}
\end{array}\right) .
\end{aligned}
$$

Proceeding with the $1 / N$ expansion as in Section 3, after rescaling

$$
\mathrm{g} \rightarrow \frac{1}{\sqrt{N}} \mathrm{~g} ; \mathrm{m} \rightarrow \sqrt{\mathrm{N}} \mathrm{m}
$$

the effective potential in the limit of vanishing momenta $\left(q^{2} \rightarrow 0\right)$ is given by the $O\langle N\rangle$ terms:

$$
\frac{1}{N} V_{e f f}(\bar{s}, \bar{D})=-\frac{1}{2} \bar{D}^{2}+\frac{1}{8 \pi^{2}} \sum_{n} n_{\nu} \operatorname{Tr} \int d^{2} q\left[\log \pi_{B}\left(q^{2}\right)-\log \pi_{F}\left(q^{2}\right)\right]_{4.6)}
$$

where $\pi_{B}$ and $\pi_{F}$ are the inverses of $B\left(q^{2}\right)$ and $F\left(q^{2}\right)$, evaluated at $s=\sqrt{\mathrm{N}} \mathrm{s}, \mathrm{D}=\sqrt{\mathrm{N}} \overline{\mathrm{D}}, \mathrm{u}=0$.

Taking the derivative with respect to $\bar{D}$, we find for the maximum:

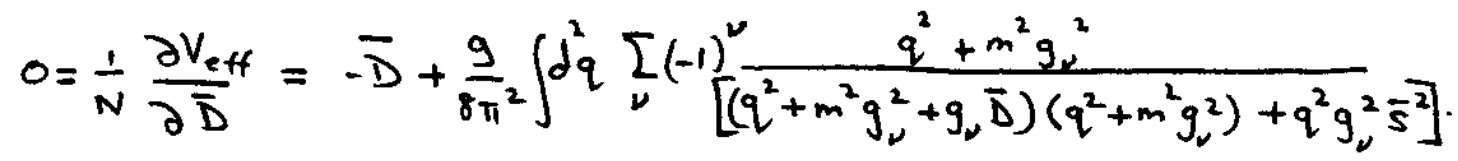

For $\vec{D}=0$ this becomes

$$
\left.\stackrel{\dot{N}}{\frac{\partial V_{e f t}}{\partial \bar{D}}}\right|_{\bar{D}=0}=\frac{9}{8 \pi} \log \left(\frac{g_{1}^{2}}{g_{2}^{2}}\right)
$$


This is non-zero if $\mathrm{g}_{1}^{2} \neq \mathrm{g}_{2}^{2}$, hence the maximum of $V_{\text {eff }}$ occurs at $\bar{D} \neq 0$ and supersymmetry is broken. To check that there are no pathologies, such as singular supersymmetric points or a maximum at infinity, we investigate the qualitative behaviour of $V_{\text {eff }}$ as a function of $\bar{s}$. Without loss of generality we can choose $g>0, n_{1}<n_{2}$. According to the discussion in Section 2, Eq. (4.7) has a single positive solution $\bar{D}(\bar{s})$. We may write

$$
V_{e f f}(\bar{s})=\int_{0}^{\bar{D}(\bar{s})} d \bar{D} \frac{\partial V_{e f f}}{\partial \bar{D}} .
$$

It is then possible to see that $v_{\text {eff }}(\bar{s})$ is a monotonic function of $\bar{s}^{2}$, which increases from a positive value at $\bar{s}^{2}=0$ to a finite value at $\bar{s}^{2}=\infty$. Thus $\vec{s}^{2}=0$ in the vacuum and supersymmetry is broken.

It also follows from (4.9) that

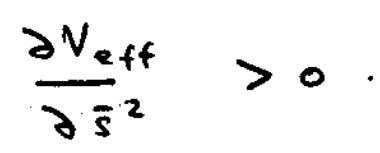

This implies that $s$ acquires a mass. However, it is not simply related to this derivative since there are finite wave function renormalizations. At the same time, the fermionic partner of $s$ should be massless, being the Goldstone fermion of broken supersymmetry. This has been confirmed by the calculations presented in Ref. 17).

It was also shown there that quantum corrections give rise to kinetic terms for the singlet fields, which were originally introduced as Lagrange multipliers. Here, they become just renormalizations of the terms already present in the classical Lagrangian. An advantage of including the kinetic terms from the beginning is that the theory is manifestly finite according to the criteria of section 2: TrV" $=0$. In Ref. 17) the effective Lagrangian for the singlet fields, obtained from performing the functional integral over the $O(N)$ vector fields, was finite, but we have not excluded divergences in higher order terms in $1 / \mathrm{N}$.

\section{5. - BEYOND TWO DIMENSIONS}

We can summarize the results obtained in the preceding sections as follows. Via the $1 / \mathrm{N}$ expansion we have demonstrated the spontaneous breaking of supersymmetry in finite two-dimensional field theories. In itself, this is not surprising since supersymmetry breaking can occur in ordinary perturbation theory. What, to us, is remarkabie is that 
1) supersymmetry breaking is facilitated if the theory is finite;

2) supersymmetry can be broken by an auxiliary component of a composite supermultiplet acquiring a non-zero vacuum expectation value; in this case the Goldstone fermion is also composite.

These observations are particularly relevant for $1 / \mathrm{N}$ expandable models since these models usually exhibit improved convergence in $1 / \mathrm{N}$ perturbation theory. In linearizing such theories auxiliary fields are introduced. These fields can represent composite states if they become dynamical in a condensed phase of the model. Thus, the $1 / \mathbb{N}$ expansion seems especially suited to establish this type of dynamical supersymetry breaking.

In view of this, it is of great interest to try to extend our analysis beyond the two-dimensional case. As a first step we consider the model discussed in Section 4 in three dimensions. In three dimensions the multiplet structure is the same as in two dimensions. The procedure is as discussed in Section 4 and Ref. 17), and results in an effective action, for the singlet fields, which is still finite in the large $\mathrm{N}$ limit. We have no proof of complete finiteness in this case and higher order corrections in $1 / N$ may well necessitate infinite renormalization.

In three dimensions, (4.7) now gives

$$
\left.\frac{1}{N} \frac{\partial V_{\text {ett }}}{\partial \bar{D}}\right|_{\bar{D}=0}=\frac{9^{2}}{8 \pi}\left(\frac{1}{n_{1}}-\frac{1}{n_{2}}\right) A\left(\bar{s}^{2}\right),
$$

where $A\left(\bar{S}^{2}\right)$ is a positive definite function of $\bar{s}^{2}$, with asymptotic behaviour

$$
A\left(\bar{s}^{2}\right)=\sqrt{\dot{s}^{2}}\left[1+O\left(\frac{m^{2}}{\dot{s}^{2}}\right)\right] \text {. }
$$

Again, for $n_{1} \neq n_{2}, \bar{D}=0$ is not a maximum of $v_{\text {eff }}$ and thus supersymmetry is broken. Also, the effective potential grows indefinitely for $\bar{s}^{2} \rightarrow \infty$, indicating that there is no dangerous extremum of the potential for large $\bar{s}^{2}$.

From this example we infer that the mechanism for supersymmetry breaking discussed here is not peculiar to two dimensions. Finiteness in the large $N$ limit also still holds. Hence, this three-dimensional model supports the arguments presented above.

Finally, we turn our attention to four-dimensional theories. Minimal supersymmetry in four dimensions is larger than minimal supersymmetry in two or three dimensions, containing four Hermitean supercharges instead of two. The larger 
supersymmetry imposes a restriction on the action which is crucial to the possibility of supersymmetry breaking by quantum corrections. That is, quantum corrections do not give rise to linear terms in the auxiliary fields of chiral multiplets to any finite order in perturbation theory. This is true for the finite as well as the infinite part. As we have seen, this is not the case in two or three dimensions. A term linear in the auxiliary field of the vector miltiplet may be generated in four dimensions, but this does not give rise to supersymmetry breaking ${ }^{22)}$. Since linear $F$ terms are not generated by quantum corrections in four dimensions, then $F=0, \phi=\phi_{0}$ remains a solution to $\partial V / \partial F=0$, if this was the solution at the tree level. Since quantum corrections do not generate $F$ independent terms either, $V$ remains zero at this point. Then, according to the argument presented in Section 2, the second derivative of $V$ with respect to the auxiliary fields remains negative, at least to first order in perturbation theory or $I / N$ expansion. Thus $\phi_{0}$ remains a supersymmetric global minimum of the effective potential.

We do not know if the second derivative of the effective potential with respect to the auxiliary fields remains negative when higher order corrections are included. However, we believe that the potential is positive definite in consistent supersymetric theories. This implies that the point $F=0, \phi=\phi_{0}$ remains a global supersymmetric minimum.

We would like to mention another difference between finite and non-finite, renormalizable theories. If infinite counterterms are necessary, then the proof in Section 2 that the second derivative of the one-loop effective potential is negative could go wrong. Then, even starting out with unbroken supersymmetry and a unique minimum at the tree level, quantum corrections could create another minimum, which is actually lower than the original, supersymmetric one. This seems to contradict the positivity of energy in supersymmetric theories. Such a situation therefore requires states with negative norm which do not decouple from physical matrix elements. We reject this possibility as being pathological.

An example of this is provided by the model of Ref. 9). In this $1 / N$ expandable scalar theory, supersymmetry is broken in that the minimum of the potential is not at zero. The potential can have a local, negative minimum and another branch which is unbounded below. A closer analysis reveals that the model indeed has both tachyons and states with negative norm ${ }^{10)}$. Clearly, the true vacuum structure must be very different from the naive one. 
The argument that radiative corrections do not break supersymmetry in ordinary perturbation theory can, in principle, be extended to the $1 / \mathbb{N}$ analysis. Since the $1 / \mathrm{N}$ expansion is essentially a resummation of perturbation theory, it seems that linear terms in the auxiliary fields are not generated. There is a loophole in this argument, though it is a remote possibility. Each term in the $1 / \mathrm{N}$ expansion is an infinite sum of ordinary Feynman diagrams. Whilst each diagram does not contain a linear $F$ term, to prove that the infinite sum of such diagrams does not contain a term linear in $F$ requires extra assumptions, for instance uniform convergence of the first derivative of the series. If the convergence is non-uniform then the summation might generate a linear $F$ term.

Finally we can envisage another way in which supersymmetry may perhaps be broken in four dimensions via the $1 / \mathrm{N}$ expansion. If an auxiliary supermultiplet acquires dynamics, i.e., $F^{2}$ terms are generated by quantum corrections, then supersymmetry might be broken at the tree level of the effective theory. This is what we found in Section 4 and Ref. 17), and advocated at the beginning of this section. In supersymmetric theories the right order of analysis might then be to look for condensates first, and then for supersymmetry breaking.

\section{ACKNOWLEDGMENTS}

We wish to thank Paolo Di Vecchia for stimulating discussions on the model described in Section 3. 


\section{REFERENCES}

1) J. Iliopoulos and B. Zumino, Nucl. Phys. B76 (1974) 310.

2) M.T. Grisaru and W. Siegel, Caltech preprint CALT 68-892 (1982);

M.B. Green, L. Brink and J. Schwartz, Caltech preprint CALT 68-880 (1981).

3) S. Ferrara and B. Zumino, Nucl. Phys. B79 (1974) 413;

L.V. Avdeev, O.V. Tarasov and A.A. Vladimir, Phys. Lett. 96B (1980) 94 ;

M.T. Grisaru, M. Rocek and W. Siegel, Phys. Rev. Lett. 45 (1980) 1063;

W.E. Caswell and D. Zanon, Nucl. Phys. Bl82 (1981) 125.

4) For a review of supergravity with many references, see:

P. van Nieuwenhuizen, Phys. Rep. 68C (1981) no. 4.

5) E. Gildener and S. Weinberg, Phys. Rev. D13 (1976) 3333;

E. Gildener, Phys. Rev. D14 (1976) 1667.

6) W. Lang, Nucl. Phys. B114 (1976) 123;

M.T. Grisaru, M. Rocek and W. Siegel, Nucl. Phys. B159 (1979) 429.

7) M.T. Grisaru, Proc. 2nd Europhysics Study Conference on Unification of Fundamental Interactions, eds. J. Ellis and S. Ferrara (Erice, 1981).

8) E. Witten, Nucl. Phys. 8185 (1981) 513;

P. Salomonson and J.W. van Holten, Nucl. Phys. B196 (1982) 509;

9) D. Zanon, Phys. Lett. 104B (1981) 127.

10) P. Salomonson, CERN preprint TH-3281 (1982).

11) E. Cremmer, S. Ferrara, B. Julia, L. Girardel10, J. Scherk and P. van Nieuwenhuizen, Phys. Lett. $79 B$ (1978) 231;

M. Sohnius and P. West, Kings' College preprint (1982).

12) J.H. Schwartz in "Supergravity", eds. P. van Nieuwenhuizen and D.Z. Freedman (North Holland, 1979) and references therein.

13) J. Ellis, Proc. 2nd Europhysics Study Conference on Unification of Fundamental Interactions, eds. J. Ellis and S. Ferrara (Erice, 1981) and references therein.

J.P. Derendinger, S. Ferrara and C.A. Savoy, Nucl. Phys. Bl88 (1981) 77 ;

14) B. de Wit and H. Nicolai, Phys. Lett. 108B (1982) 285.

15) A. D'Adda, P. Di Vecchia and M. Lüscher, Nucl. Phys. B146 (1978) 63; ibid. B152 (1979) 125;

E. Witten, Nucl. Phys. BI49 (1979) 285.

16) O. Alvarez, Phys. Rev. DI7 (1978) 1123.

17) A.C. Davis, P. Salomonson and J.W. van Holten, CERN preprint TH-3256 (1982).

18) See also:

L. Azvarez-Gaumé, D.Z. Freedman and M.T. Grisaru, Harvard University preprint HUTMP 81/BIll.

19) A similar observation has been made independently before us by:

S. Browne, Phys. Lett. 59B (2975) 253.

20) For a general discussion see, for example:

P. Fayet and S. Ferrara, Phys. Rep. 32C (1977) no. 5.

21) S. Coleman, Comm. Math. Phys. 31 (1973) 259.

22) B.A. Ovrut and J. Wess, Karlsruhe preprint (1981);

W. Fischler, H.P. Nilles, J. Polchinski, S. Raby and L. Susskind, Phys. Rev. Lett. 47 (1981) 757 . 

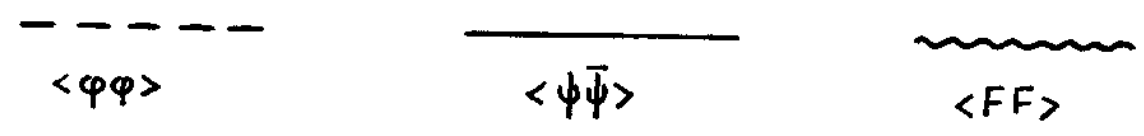

Fig. I
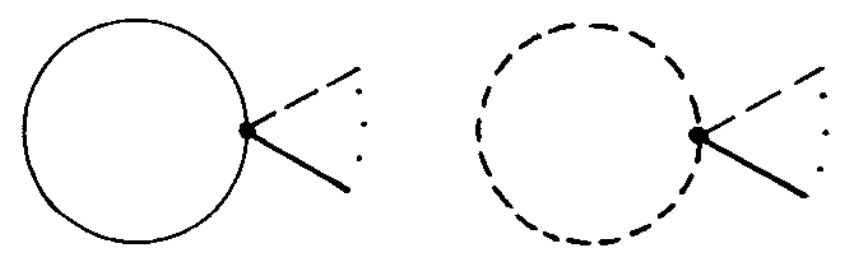

Fig. 2
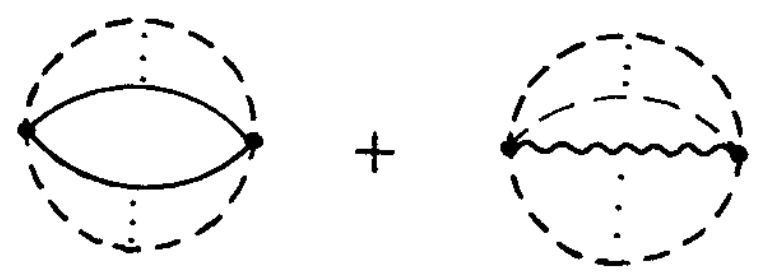

Fig. 3

\section{FIGURE CAPTIONS}

Figure 1 : Definition of the propagators.

Figure 2 : Divergent diagrams with $\mathrm{V}=1$.

Figure 3 : Divergent diagrams with $V=2$. 
